\title{
La collection de papyrus et d'ostraca de la bibliothèque universitaire de Leipzig
}

Reinhold Scholl (traduction Frédéric Blin)

\section{Q OpenEdition}

1 Journals

Édition électronique

URL : https://journals.openedition.org/rbnu/3330

DOI : 10.4000/rbnu.3330

ISSN : 2679-6104

Éditeur

Bibliothèque nationale et universitaire de Strasbourg

Édition imprimée

Date de publication : 1 novembre 2010

Pagination : 58-63

ISSN : 2109-2761

\section{Référence électronique}

Reinhold Scholl (traduction Frédéric Blin), « La collection de papyrus et d'ostraca de la bibliothèque universitaire de Leipzig », La Revue de la BNU [En ligne], 2 | 2010, mis en ligne le 01 novembre 2010, consulté le 08 août 2021. URL : http://journals.openedition.org/rbnu/3330 ; DOI : https://doi.org/ $10.4000 /$ rbnu. 3330

\section{(c) (i) (8)}

La Revue de la BNU est mise à disposition selon les termes de la Licence Creative Commons Attribution - Pas d'Utilisation Commerciale - Partage dans les Mêmes Conditions 4.0 International. 


\section{La collection de papyrus et d'ostraca de la bibliothèque universitaire de Leipzig}

\section{Les débuts de la collection}

La collection de papyrus et d'ostraca de la bibliothèque de l'université de Leipzig a été initiée en 1902. Trois institutions étaient alors impliquées : le ministère d'Etat de Saxe pour les cultes et l'enseignement public, la Société royale des sciences de Saxe ainsi que la Fondation Albrecht de Leipzig. Celles-ci permirent de financer à l'été 1902 l'acquisition de papyrus en provenance d'Egypte, dont une majorité écrits en grec. Une charte d'exploitation des papyrus fut alors établie, visant à permettre un accès libre aux documents.

Mais des papyrus étaient déjà parvenus jusqu’à Leipzig avant la fondation de cette société. Parmi ceux-ci figure le célèbre Papyrus Ebers, qui avec ses 18,63 mètres est le rouleau le plus long et le mieux conservé se rapportant à la médecine de l'Egypte ancienne au $\mathrm{XVI}^{e}$ siècle avant J.-C. L'égyptologue leipzigois Georg Ebers avait acheté le rouleau sur place à l'hiver 1872/1873 et l'avait confié à la bibliothèque universitaire. Le rouleau conservé dans son intégralité fut découpé en 29 morceaux; chacun d'entre eux fut ensuite mis sous verre et encadré. Un luxueux fac-similé de ce papyrus fut ensuite publié en $1875^{1}$.

Le célèbre théologien Konstantin von Tischendorf, à qui Leipzig doit aussi quelques feuillets du Codex Sinaiticus, avait lui aussi ramené à Leipzig des papyrus de ses voyages en Egypte, et les avait donnés à la bibliothèque universitaire. Ces papyrus ont été publiés par Carl Wessely ${ }^{2}$. Les originaux ont été dans ce cas collés dans un recueil, où ils se trouvent encore aujourd'hui.

\section{Le cartel des papyrus}

Mais la majorité des papyrus de Leipzig furent acquis grâce au " cartel allemand des papyrus " (Papyruskartell), qui fut fondé en 1902 par divers musées, instituts universitaires et personnes privées et auquel appartenait aussi l'université de Strasbourg et sa bibliothèque. L'objectif de ce cartel était l'achat en commun de papyrus en Egypte, afin de ne pas faire monter les prix par des enchères concurrentes. Les membres recevaient ensuite une courte description des différents lots, sur lesquels il était ensuite possible d'enchérir. Le cartel comportait deux départements, l’un pour les papyrus littéraires, l'autre pour les papyrus documentaires. Leipzig était membre des deux départements. Prix Nobel de littérature en 1902 pour son Histoire romaine, l'historien de l'Antiquité Theodor Mommsen versa une part importante de sa récompense pour permettre d'enrichir la collection de papyrus de Leipzig.

Dans les années 1930 furent de nouveau achetés quelques papyrus, grâce au soutien financier de la Société des amis de l'université de Leipzig.

La première description scientifique fut éditée à la mémoire de Theodor Mommsen par l'historien du droit leipzigois Ludwig Mitteis et par l'historien de l'Antiquité et fondateur de la papyrologie scientifique Ulrich Wilcken, qui occupa la chaire d'histoire ancienne à Leipzig entre 1906 et $1912^{3}$. Certains textes furent ensuite publiés séparément dans des revues spécialisées. 


\section{Prix Nobel de littérature en 1902 pour son Histoire romaine, l'historien de l'Antiquité Theodor Mommsen versa une part importante de sa récompense pour permettre d'enrichir la collection de papyrus de Leipzig}

\section{Les aléas d'une collection}

A la restauration des papyrus succédaient l'inscription à l'inventaire et un catalogage sommaire, pour environ 600 pièces à l'époque. Après la Seconde Guerre mondiale, c'est le Nestor de la papyrologie allemande, Wilhelm Schubart, alors âgé de 73 ans, qui fut chargé de la chaire d'histoire ancienne. Mais les points forts de l'enseignement et de la recherche ne résidaient pas dans le domaine de la papyrologie, et au cours de la période suivante, l'exploitation des sources directes retomba à l'arrière-plan. C'est ainsi qu'il n'y eut ni personnel scientifique ni personnel de conservation dédié à la collection de papyrus, et les documents non encore traités restèrent dans l'état de leur achat par le Papyruskartell et commencèrent à se détériorer.

La Seconde Guerre mondiale eut comme conséquence certaines pertes et destructions. Le Papyrus Ebers était au départ conservé dans la chambre forte d'une banque de Leipzig, avant d'être évacué vers le château de Rochlitz sur la Mulde, à 60 kms au sudouest de Leipzig. On raconte qu'il fut redécouvert à la fin de la guerre dans une niche de chien. Des 29 parties conservées avant la guerre, les parties XIII (colonnes 48, 49), XXIV (col. 80, 81, 82) et XXIX (col. $99,100,101,102,103,104,105)$ furent totalement détruites, d'autres furent endommagées. Nous ne connaissons pas le sort des trois dernières parties, mais nous ne désespérons pas de les voir réapparaître un jour. On déplore en outre la perte d'une quarantaine d'autres papyrus.

A l'époque, le tome 1 du catalogue des papyrus de Leipzig n'a jamais été suivi d'un tome 2, mais certains textes ont toutefois été édités avec le temps par des papyrologues extérieurs.

\section{La collection aujourd'hui}

L'auteur de cet article prit la responsabilité de la collection en 1993. A l'été 1994, tous les papyrus furent transférés de leurs caisses métalliques vers des boîtes en carton neutre et conservés pour partie entre des feuilles de papier neutre. Grâce à cette opération, on obtint pour la première fois un aperçu approximatif de la collection : on recensa environ 5 ooo papyrus et fragments de papyrus, ce qui fait de la collection de Leipzig un fonds de première importance. La très grande majorité des papyrus est grecque, mais on y trouve également des papyrus hiératiques, démotiques, latins, coptes et arabes. En outre, le papyrus n'est pas le seul support d'écriture : parchemin et papier figurent également dans la collection.

Celle-ci compte aussi 1644 ostraca. Une partie arriva à l'été 1902 à Leipzig ; 724 sont écrits en grec, 292 en démotique, 628 en copte. Très peu sont bilingues. Seize ostraca grecs furent publiés sous le titre P. Lips. I. Certains ont été édités au fil du temps par Ulrich Wilcken et par le directeur de la collection de papyrus à Jena, Fritz Uebel. Des pièces démotiques et coptes furent décrites de manière isolée dans diverses revues au cours des années 1990 et 2000. Mais 1500 ostraca environ attendent encore d'être étudiés et publiés, tandis que grâce à un soutien financer de la fondation Fritz Thyssen, Ruth Duttenhöfer a pu réaliser un volume sur les papyrus de Leipzig, publié en 2002 sous le titre P. Lips. II ${ }^{4}$.

Les papyrus et ostraca de Leipzig entrèrent eux aussi dans une nouvelle ère avec l'arrivée des nouveaux médias, de la numérisation et de l'internet. Le programme " Papyrus Halle-Jena-Leipzig " fut conçu et mis en œuvre grâce au financement de la Fondation allemande pour la recherche (Deutsche 


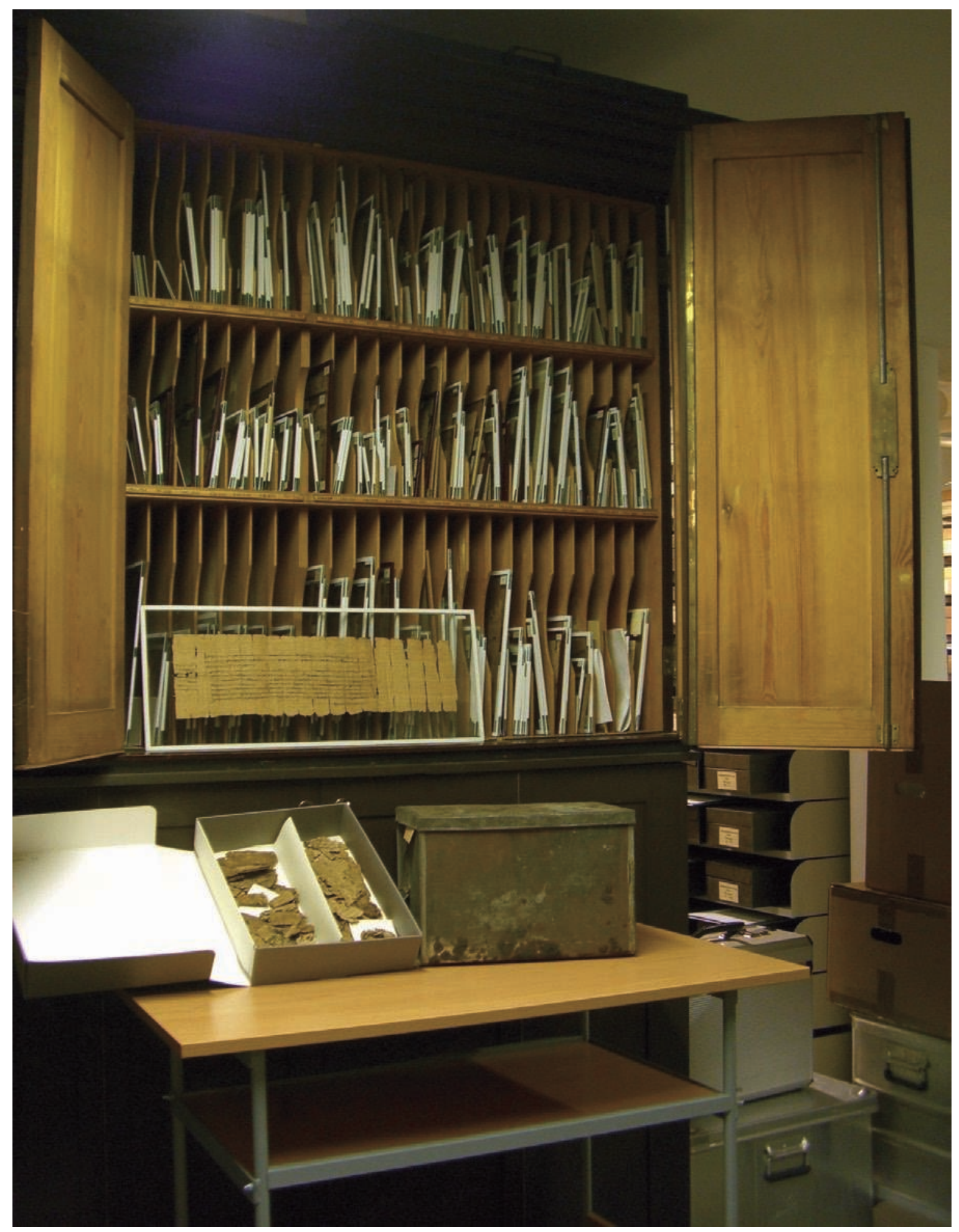

Armoire à papyrus de la bibliothèque universitaire de Leipzig 
Forschungsgemeinschaft - DFG). Dans ce projet mené sous la coordination de l'université de Leipzig, les collections de papyrus des universités de Halle, Jena et Leipzig sont depuis 2003 restaurées, numérisées, microfilmées, décrites suivant des normes compatibles avec d'autres projets internationaux, et enfin mises en ligne sur Internet (http://papyri.uni-leipzig.de).

\section{Perspectives}

Depuis 2009, ce sont les fonds d'ostraca qui sont traités de même manière dans le cadre d'un projet de la DFG. A la prise de vue en infrarouge s'ajoute pour quelques objets choisis une étude plus approfondie, suivant les techniques les plus modernes cherchant à obtenir la meilleure image tout en préservant le document. Ces études sont effectuées par l'Institut de minéralogie, cristallographie et de science matérielle de l'université de Leipzig. Les images et les nombreuses données de mesure seront consultables à l'adresse internet évoquée ci-dessus, et conduiront sans doute à de nouvelles recherches et découvertes dans les domaines de l'histoire ancienne et de la papyrologie.

Dans un autre projet financé par la DFG, ce sont les collections de papyrus conservées en Allemagne qui ont été et qui seront mises en ligne sur un portail internet commun, qui permettra une recherche et une présentation harmonisées des résultats sur la Toile. Les collections, tout comme les banques de données, pourront être interrogées en temps réel. Grâce à ce portail, les différences technologiques et de contenus scientifiques des diverses bases de données seront effacées. A côté des collections actuellement prises en compte (Bonn, Erlangen, Giessen, Halle, Heidelberg, Jena, Cologne, Leipzig, Trèves et Würzburg...) s'ajouteront prochainement Berlin et d'autres ensembles. Il serait souhaitable qu'une recherche dans la collection strasbourgeoise soit également possible via le portail des papyrus (www. papyrusportal.net). Car ainsi, ce serait sur l'ensemble des anciens membres du cartel que l'on pourrait faire des recherches simultanément. Les archives du cartel sont encore presque complètement conservées à Strasbourg, quand par exemple à Leipzig elles ont toutes disparu. Peut-être serait-il envisageable de numériser ces archives strasbourgeoises afin de les rendre disponibles aux chercheurs sur Internet. Si les autres collections diffusaient ensuite elles aussi leurs archives et les documents relatifs à leur activité au sein du cartel, nous pourrions ainsi identifier des relations entre documents et reconstituer virtuellement des ensembles autrefois cohérents, mais qui avec le temps ont été malencontreusement séparés et dispersés.

\section{Collections virtuelles}

Un exemple de lien étroit entre les collections de Leipzig et de Strasbourg réside par exemple dans le fonds d'archives d'Aurelios Kyros, nyktostratège (c'est-à-dire chef de la garde de nuit) à Hermopolis à la fin du IV ${ }^{e}$ siècle après J.-C., fonds qui d'après nos connaissances actuelles est divisé entre les deux collections. Un papyrus, qu'Ernst Kornemann avait acheté en 1902 à Eschmunein, se trouve en outre à Giessen. Seule une petite partie des textes des deux collections est aujourd'hui publiée. On peut trouver une présentation des textes identifiés de ce fonds, faite par Daniela Colomo (à l'adresse http://papyri-leipzig.dl.uni-leipzig.de/content/main/ archiv_aurelios_kyros.xml). Une belle et ambitieuse entreprise serait de publier de manière unifiée ce fonds aujourd'hui divisé en deux collections.

Mais ce n'est pas uniquement dans le domaine des papyrus qu'on trouve des collections éclatées entre Strasbourg et Leipzig. C'est ainsi que les ostraca de Strasbourg $\mathrm{n}^{\circ}$ 662-771 forment avec plusieurs douzaines d'ostraca de Leipzig et de la Bodleian Library d'Oxford un ensemble important, remontant au $\mathrm{II}^{\mathrm{e}}$ siècle après J.-C., et renvoyant à un homme dénommé Horon, fils de Makron. Ces exemples témoignent des liens étroits existant entre les collections de Strasbourg et de Leipzig.

Outre la numérisation et la diffusion des métadonnées $^{5}$ sur Internet, un autre objectif intrinsèque à toute collection de papyrus est l'édition proprement dite, c'est-à-dire le déchiffrage de l'intégralité des textes, leur transcription, leur traduction, ainsi que la rédaction d'un commentaire philologique et historique les resituant dans leur contexte propre. A Leipzig, les personnes employées dans le cadre de ces projets travaillent aujourd'hui à l'édition du volume III des papyrus. En parallèle, une 
exposition s'est tenue à la bibliothèque universitaire de Leipzig du 18 juin au 26 septembre 2010, sous le titre " Enterré, perdu, retrouvé, étudié. Les trésors des papyrus de Leipzig ". Un catalogue de l'exposition est également publié ${ }^{6}$, et d'autres projets sont actuellement en cours.

Reinhold Scholl (traduction Frédéric Blin)

\section{Notes :}

1 - Papyros Ebers. Das hermetische Buch über die Arzneimittel der alten Ägypter in hieratischer Schrift. Herausgegeben, mit Inhaltsangabe u. Einleitung versehen von Georg Ebers. Mit hieroglyphischlateinischem Glossar von Ludwig Stern ; Bd. 1-2 ; Bd.1. Einleitung und Text, Tafel I-LXIX ; Bd.2. Glossar und Text, Tafel LXX-CX, Leipzig, 1875

2- Carl Wessely, Die griechischen Papyri der Leipziger Universitätsbibliothek, Leipzig, 1885 (Verhandlungen der königlichen Sächsischen Gesellschaft der Wissenschaften 37, S. 237-75, Nr. 1-35)

3 - Griechische Urkunden der Papyrussammlung zu Leipzig. I. Bd. Mit Beiträgen von Ulrich Wilcken, hrsg. von Ludwig Mitteis, Leipzig, 1906

4- Griechische Urkunden der Papyrussammlung zu Leipzig (P.Lips. II), hrsg. von Ruth Duttenhöfer mit einem Beitrag von Reinhold Scholl (Archiv für Papyrusforschung und verwandte Gebiete, Beiheft 10), München und Leipzig, 2002

5- Données informatiques permettant, une fois la numérisation faite, de décrire un document.

6-Vergraben, verloren, gefunden, erforscht. Papyrusschätze in Leipzig, Katalog zur Ausstellung Universitätsbibliothek Leipzig 18. Juni bis 26. September 2010, hrsg. von Reinhold Scholl (Schriften aus der Universitätsbibliothek ; 20), Leipzig, 2010 